\title{
Diagnosis and prognosis of right ventricular infarction
}

Sir,

The important paper by Dr Rodrigues and colleagues $(1986 ; 56: 19-26)$ showed that in a series of 51 patients with acute infarction of the inferior myocardial wall, right ventricular ejection fraction was depressed in all cases, while pyrophosphate scintigraphy was positive in $25(\simeq 50 \%)$ of the 51 patients. Moreover, severe right ventricular dysfunction was taken to be a right ventricular ejection fraction of $<0.25$ and $50 \%$ of their patients fell into this category. They also argued that this stricter criterion (right ventricular ejection fraction $<0.25$ ) enhances the specificity of radionuclide ventriculography in the diagnosis of right ventricular involvement.

We should like to make the following comments:

(a) It is surprising that they found a clear cut depression of right ventricular ejection fraction in all patients; this finding does not accord with the pathoanatomical ${ }^{1}$ and radionuclide ${ }^{2-4}$ reports and it suggests incorrect selection of the patient population. Did they exclude patients with chronic pulmonary obstructive disease, valvar heart disease, or right bundle branch block?

(b) Rodrigues et al obtained negative pyrophosphate scans in about half of their patients. If this finding is compared with that in $(a)$, it follows that half of their patients showed right ventricular dysfunction in the absence of scintigraphic, and perhaps also clinical and electrocardiographic, findings consistent with right ventricular necrosis.

(c) Using a right ventricular ejection fraction $<0.25$ as the criterion for right ventricular dysfunction Rodrigues et al reported involvement of the right ventricle in $50 \%$ of patients. What then, was the meaning of the lower limit $(0.53)$ of normal range for right ventricular ejection fraction?

(d) Garty et al emphasised that regional wall motion abnormalities are the most constant finding with the greatest sensitivity and specificity in the diagnosis of right ventricular infarction. ${ }^{4}$ We and our coworkers demonstrated a statistically significant correlation between depressed right ventricular ejection fraction and the presence of regional wall motion abnormalities of the right ventricle in a series of patients with acute infarction of the inferior myocardial wall. ${ }^{5}$ Analysis of right ventricular regional wall motion was not reported by Rodrigues et al.

(e) Rodrigues et al did not report left ventricular ejection fractions in the acute phase of infarction (days 2 to 4 ) nor give details of treatment.

(f) Could Dr Rodrigues and colleagues explain why mean right ventricular ejection fraction was 0.21 in the 14 patients without clinical features of right ventricular dysfunction?

B Palagi,

R Picozzi,

R Baroffio,

Servizio di Medicina Nucleare and

Servizio di Cardiologia ed Unità Coronarica,

Ospedale di Saronno,

21047 Saronno (VA), Italy.

\section{References}

1 Wartman WB, Hellerstein HK. The incidence of heart disease in 2000 consecutive autopsies. Ann Intern Med 1948;28:41-65.

2 Sharpe DN, Botvinick EH, Shames DM, et al. The non-invasive diagnosis of right ventricular infarction. Circulation 1978;57:483-90.

3 Wackers FJ, Lie KI, Sokale EB, Res J, van der Schoot JB, Durrer D. Prevalence of right ventricular involvement in inferior wall myocardial infarction assessed with myocardial imaging with thallium-201 and technetium-99m pyrophosphate. Am J Cardi, 1978;42:358-62.

4 Garty I, Barzilay J, Bloch L, Antonelli D, Koltum B. The diagnosis and early complications of right ventricular infarction. Eur J Nucl Med 1984;9:453-60.

5 Palagi B, Baroffio R, Picozzi R, et al. Radionuclide 3 assessment of right ventricular involvement in inferior acute myocardial infarction:clinical correlations and in-hospital follow-up. Eur J Nucl Med 1985; 10:235-40.

This letter was shown to the authors who reply as follows:

Sir,

We thank Dr Palagi and colleagues for their interest in our paper and for their comments. They poseco a number of questions which we will try to answer succinctly.

Patient selection is important; our patients had 0 been admitted to our coronary care unit and over $90 \%$ had sustained transmural myocardial $\stackrel{\mathbb{\Phi}}{\varrho}$ infarction. We excluded patients with chronic $\stackrel{\mathbb{Q}}{\varrho}$ obstructive pulmonary disease or valvar heart dis- $\bar{O}$ ease. We have previously reported that after anterior 